\title{
On the computation of discrete Fourier transform using Fermat number transform
}

\author{
Wan-Chi Siu, AP(HK), M.Phil., C.Eng., M.I.E.R.E., Mem.I.E.E.E., and A.G. \\ Constantinides, B.Sc.(Eng.), Ph.D., C.Eng., M.I.E.E., Sen.Mem.I.E.E.E.
}

Indexing terms: Signal processing, Discrete Fourier transforms, Fermat number transforms

\begin{abstract}
In the paper the results of a study using Fermat number transforms (FNTs) to compute discrete Fourier transforms (DFTs) are presented. Eight basic FNT modules are suggested and used as the basic sequence lengths to compute long DFTs. The number of multiplications per point is for most cases not more than one, whereas the number of shift-adds is approximately equal to the number of additions in the WinogradFourier-transform algorithm and the polynomial transform. Thus the present technique is very effective in computing discrete Fourier transforms.
\end{abstract}

\section{Introduction}

The introduction of the fast Fourier transform (FFT) algorithm by Cooley and Tukey $[1,2]$ in 1965 allowed a tremendous saving in calculating effectively the discrete Fourier transform (DFT). This algorithm reduces the number of real multiplications for a direct DFT computation from $4 N^{2}$ to $2 N \log _{2} N$, while the number of real additions is reduced from $2 N^{2}$ to $3 N \log _{2} N$ for $N$-point DFTs. The major disadvantages of the FFT algorithm are that (i) it still requires quite a large number of multiplications and (ii) the number of real multiplications per point is almost the same for both real and complex input data. This last point makes the FFT very inefficient for the purpose of calculating the DFT of real input data. In 1975, Winograd [3] showed that the minimum number of multiplications required to compute the circular convolution of two length- $N$ sequences is $2 N-K$, where $K$ is the number of divisors of $N$ including 1 and $N$. Agarwal and Cooley [4], Winograd [5] and Kolba and Parks [6] made use of Rader's theorem [7] on DFT with prime transform length to construct their algorithms for the computation of DFT. Compared with conventional FFT methods, the Winograd-Fourier-transform algorithms require only onehalf to one-third of the number of multiplications needed for conventional FFT, with a slightly larger number of additions per point. In 1977, Nussbaumer [8] first suggested the use of polynomial transform over the field of polynomials for computing two-dimensional convolutions. This approach led to the development of fast algorithms [9-11] for computing DFTs using polynomial transforms. The method requires two to four real multiplications per point for complex input data. For the computation of real input data, both the Winograd-Fourier-transform algorithm and polynomial transform require in fact only-half of the operations needed for the case of complex data. Reed and Truong $[12,13]$ proposed a technique for the computation of discrete Fourier transforms, based on Winograd's method in combination with Mersenne prime number theoretic transforms. This hybrid algorithm requires fewer multiplications than either the standard FFT or Winograd's more conventional algorithm.

Paper 2920F, first received 9th May 1983 and in revised form 22nd September 1983 The authors are with the Department of Electrical Engineering, Imperial College of Science \& Technology, London SW7 2BT, England. Wan-Chi Siu is on leave from the Department of Electronic Engineering, Hong Kong Polytechnic, Hung Hom, Hong Kong
However, a very large number of additions are required and the number of multiplications per point is still relatively large (about 1.33 to 3.49 multiplications per point).

Recently Siu and Constantinides [14] have shown that the number of multiplications for DFT computations can be reduced further by using number theoretic transforms (NTT) [15-18]. The total number of real multiplications in this case in fact reduces to $(P-1)$ for computing a $P$-point DFT, where $P$ is a prime number (or $1-1 / P$ multiplications per point). Of the various versions of the NTT proposed, the Fermat number transform (FNT), which has been thoroughly investigated by Agarwal and Burrus [17], and the Mersenne number transform (MNT), which was introduced by Rader [16], appear to offer the greater promise vis-a-vis speed and complexity. However, if we take the number of shift-adds into account for the evaluation of the entire computational effort, it turns out that, because there is no fast algorithm (or fast Fourier transform type algorithm) for the computation of Mersenne number transforms, a large number of shift-adds have to be used for the computation of discrete Fourier transforms using MNT. Some fast Fourier transform types of algorithms can possibly be used in some pseudo-Fermat number transforms [19] and pseudo-Mersenne transforms [20]. The transform length of the Fermat number transform is highly composite (it is in fact a power of two), and hence fast Fourier transform types of algorithms can be used for the computation. In this respect, it would appear that the FNT is the most prominent candidate for the computation of DFTs. However, for actual implementation, all number theoretic transforms are limited by sequence-length constraints [21]. This limits the application of FNT to a small number of relatively short DFTs only, or indeed the application of number theoretic transform for computing the discrete Fourier transform is limited as a whole.

In this paper, we present the results of our study using Fermat number transforms to compute DFTs and propose various techniques for computing long discrete Fourier transforms.

\section{Basic Fermat-number-transform modules}

The Fermat number transform (FNT) and its inverse are defined as follows:

$$
X_{k}^{\prime \prime}=\left\langle\sum_{n=0}^{N-1} x_{n} \alpha^{n k}\right\rangle_{M^{\prime}}
$$


and

$$
x_{n}=\left\langle\frac{1}{N} \sum_{k=0}^{N-1} X_{k}^{\prime \prime} \alpha^{-n k}\right\rangle_{M^{\prime}} \quad \text { for } n, k=0,1, \ldots, N-1
$$

where $M^{\prime}=2^{2 t}+1$, a Fermat number, for $t=1,2, \ldots$, $\alpha=$ root of unity of order $N$ and is chosen to be 2 or a power of 2 for most cases, and $\alpha^{m} \neq 1,1 \leqslant m<N$.

The expression $\langle C\rangle_{M^{\prime}}$ means the residue of the number $C$ modulo $M^{\prime}$. $N$ is also a power of 2 and its maximum value is $2^{t+1}$ for $\alpha=2$. Similar to the discrete Fourier transforms, the FNT can be used to compute cyclic convolutions. Let us consider the cyclic convolution of the two sequences, $\left\{x_{n}: n=0,1, \ldots, N-1\right\}$ and $\left\{h_{n}: n=0,1, \ldots\right.$, $N-1\}$.

$$
X_{m}=\sum_{n=0}^{N-1} x_{n} h_{m-n} \text { for } m=0,1, \ldots, N-1
$$

For the computation of eqn. 3 using FNT $X_{m}$ can be found from the inverse Fermat number transform (IFNT)

$$
X_{m}=\left\langle\frac{1}{N} \sum_{k=0}^{N-1} Y_{k}^{\prime \prime} \alpha^{-m k}\right\rangle_{M^{\prime}} \text { for } m=0,1, \ldots, N-1
$$

The coefficients $Y_{k}^{\prime \prime}$ are the products of the term-by-term multiplications of the corresponding $X_{k}^{\prime \prime}$ and $H_{k}^{\prime \prime}$, and $H_{k}^{\prime \prime}$ is the FNT of the sequence $\left\{h_{n}: n=0,1, \ldots, N-1\right\}$.

Consider now the discrete Fourier transform of a real sequence $\{x(n): n=0,1, \ldots, N-1\}$

$$
Y(k)=\sum_{n=0}^{N-1} x(n) W_{0}^{n k}
$$

where $k=0,1, \ldots, N-1$ and

$$
W_{0}=\exp \left(-j \frac{2 \pi}{N}\right)
$$

Let us denote $\left\langle g^{n}\right\rangle_{P}$ as the residue of the number $g^{n}$ modulo $P$, where $g$ is a primitive root that generates all nonzero elements inside the field modulo $P$. If $N$ is a prime number, $P$ for example, eqn. 4 can be reordered $[14,7]$ in the following form:

$$
Y(0)=\sum_{n=0}^{P-1} x(n)
$$

and

$$
Y\left(\left\langle g^{k}\right\rangle_{P}\right)=X(0)+X\left(\left\langle g^{k}\right\rangle_{P}\right)
$$

where

$$
\begin{aligned}
& X\left(\left\langle g^{k}\right\rangle_{P}\right)=\sum_{n=1}^{P-1} x\left(\left\langle g^{-n}\right\rangle_{P}\right) W_{0}\left\langle g^{k-n}\right\rangle_{P} \\
& \qquad \text { for } k=1,2, \ldots, P-1
\end{aligned}
$$

Eqn. 7 represents a backward cyclic convolution of length $-(P-1)$ and can be expressed as the convolution

$$
\begin{array}{r}
\left\{x_{0}, x_{1}, \ldots, x_{P-2}\right\} \circledast\left\{W_{0}, W_{1}, \ldots, W_{(P-1) / 2-1},\right. \\
\left.W_{0}^{*}, W_{1}^{*}, \ldots, W_{(P-1) / 2-1}^{*}\right\}
\end{array}
$$

where

$$
\begin{aligned}
& x_{n}=x\left(\left\langle g^{-(n+1)}\right\rangle_{P}\right. \\
& W_{n}=W_{0}^{\left.g^{n}\right\rangle_{p}} \text { for } n=0,1, \ldots, P-2
\end{aligned}
$$

The symbols * and $\circledast$ mean complex conjugate and cyclic convolution, respectively. This cyclic convolution sum can be computed by Fermat number transform. Siu and Constantinides [14] have already pointed out that the real and imaginary parts of the number theoretic transformed results of $\left\{W_{0}, W_{1}, \ldots, W_{(P-1) / 2-1}, W_{0}^{*}, W_{1}^{*}, \ldots\right.$, $\left.W_{(P-1) / 2-1}^{*}\right\}$ are alternately zero, thereby reducing the total number of multiplications to $(P-1)$ for $P$-point DFTs.

It is also interesting to point out that, since the sequence $\{x(n): n=0,1, \ldots, N-1\}$ is real, $\left\{X\left(\left\langle g^{k}\right\rangle_{P}\right): k=1,2, \ldots\right.$, $P-1\}$ can be put in the form of

$$
\begin{aligned}
\left\{X\left(\left\langle g^{1}\right\rangle_{P}\right), X\left(\left\langle g^{2}\right\rangle_{P}\right), \ldots,\right. & X\left(\left\langle g^{(P-1) / 2}\right\rangle_{P}\right), X\left(\left\langle g^{1}\right\rangle_{P}\right)^{*}, \\
& \left.X\left(\left\langle g^{2}\right\rangle_{P}\right)^{*}, \ldots, X\left(\left\langle g^{(P-1) / 2}\right\rangle_{P}\right)^{*}\right\}
\end{aligned}
$$

Hence this expression shows that, for actual implementation, only $\left\{X\left(\left\langle g^{1}\right\rangle_{P}\right), X\left(\left\langle g^{2}\right\rangle_{P}\right), \ldots, X\left(\left\langle g^{(P-1) / 2}\right\rangle_{P}\right)\right\}$ need to be computed, the other half of the $X\left(\left\langle g^{n}\right\rangle_{p}\right)$ s can be found by taking the conjugates of these values.

Notice that a Fermat number is defined as $F_{t}=2^{2 t}+1$; hence $F_{t}-1\left(=2^{2 t}\right)$ is a highly composite number or a power of 2 , and so if $F_{t}$ is chosen as the DFT length, the corresponding $\left(F_{t}-1\right)$-point cyclic convolution can be computed by an NTT (in our case FNT) with an FFT-type algorithm.

Fermat numbers up to $F_{4}$, namely $\{3,5,17,257$, $65537\}$, are all primes, and these are possible sequence lengths (i.e. $P=F_{t}$ for $t=0,1,2,3,4$ ) for the computation of discrete Fourier transform using NTT.

For example, let us take $F_{2}(=17)$ to illustrate the idea. Consider the DFT of the sequence $\{x(0), x(1), \ldots, x(16)\}$, i.e. $N=P=17$. In this case 3 is a primitive root which can be used to generate all elements inside the field modulo 17. Hence eqn. 8 becomes

$$
\begin{aligned}
& \{x(6), x(2), x(12), x(4), x(7), x(8), x(14), x(16), x(11), \\
& \quad x(15), x(5), x(13), x(10), x(9), x(3), x(1)\} \\
& \circledast\left\{W_{0}^{1}, W_{0}^{3}, W_{0}^{9}, W_{0}^{10}, W_{0}^{13}, W_{0}^{5}, W_{0}^{15}, W_{0}^{11}, W_{0}^{1 *},\right. \\
& \left.W_{0}^{3 *}, W_{0}^{9 *}, W_{0}^{10 *}, W_{0}^{13 *}, W_{0}^{5 *}, W_{0}^{15 *}, W_{0}^{1 *}\right\}
\end{aligned}
$$

This convolution sum can be computed by using a Fermat number transform. If we choose $M$, the base for modulo arithmetic in FNT, to be $F_{4}\left(2^{16}+1\right)$, the generator $\alpha$ for the transform is $2^{2}$ and of course $N=16$. On the other hand if we choose $M=F_{5}\left(2^{32}+1\right)$ then $\alpha=2^{4}$ and $N$ of course remains as 16 . In all cases, the transformed results of $\left\{W_{0}^{1}, W_{0}^{3}, W_{0}^{9}, \ldots, W_{0}^{15 *}, W_{0}^{1 *}\right\}$ have their real and imaginary parts alternately zero. Hence 16 real multiplications are required for the computation of a 17-point realinput-data DFT.

For actual implementation, not all Fermat primes that can be used as sequence lengths are attractive for the computation of DFT. For example, $F_{4}(=65537)$ is long enough (or perhaps too long) for nearly all kinds of applications, but there is no suitable Fermat number transform (with sequence length $=65537-1=65536$ ) available for such a long sequence length. In the light of this situation, it would seem that the most attractive transform lengths are $F_{1}(=5), F_{2}(=17)$ and $F_{3}(=257)$. Table 1 lists the number of real operations for four possible prime (Fermat primes) lengths and four other lengths (to be discussed below). The number of multiplications is found by

$$
M=P-1
$$

and the number of shift-adds (with slight modification for length 257 , see below) is found by [14]

$$
A=(P-1)\left[2 \log _{2}(P-1)+1\right]
$$

It can be seen from Table 1 that for length 257 DFT we have only a single choice of base $\left(F_{6}\right)$ for modulo arithmetic, and in this case the generator is $\sqrt{2}$. Agarwal and 
Table 1: Number of real multiplications and shift-adds for real data DFT computed by FNT

\begin{tabular}{lllll}
\hline $\begin{array}{l}\text { DFT } \\
\text { length }\end{array}$ & $\begin{array}{l}\text { Possible } \\
\text { modulo } \\
\text { base }\end{array}$ & $\begin{array}{l}\text { Possible } \\
\text { root of } \\
\text { unity }\end{array}$ & $\begin{array}{l}\text { Number } \\
\text { of real } \\
\text { multiplications } \\
M \text { (per point) }\end{array}$ & $\begin{array}{l}\text { Number } \\
\text { of real } \\
\text { shift-adds } \\
A \text { (per point) }\end{array}$ \\
\hline 3 & $F_{1}, \ldots, F_{8}$ & $2^{2}, \ldots, 2^{64}$ & $2(0.67)$ & $6(2.0)$ \\
5 & $F_{1}, \ldots, F_{8}$ & $2, \ldots, 2^{32}$ & $4(0.80)$ & $20(4.0)$ \\
17 & $F_{3}, F_{4}, F_{5}, F_{8}$ & $2, \ldots, 2^{8}$ & $16(0.94)$ & $144(8.5)$ \\
257 & $F_{8}$ & $\sqrt{2}$ & $256(1.00)$ & $4544(17.7)$ \\
13 & $F_{1}, \ldots, F_{8}$ & $2, \ldots, 2^{32}$ & $16(1.23)$ & $104(8)$ \\
97 & $F_{4}, F_{5}, F_{8}$ & $2,2^{2}, 2^{3}, 2^{4}$ & $128(1.32)$ & $1408(14.51)$ \\
193 & $F_{5}, F_{6}$ & $2,2^{2}$ & $256(1.33)$ & $3200(16.6)$ \\
769 & $F_{8}$ & $\sqrt{2}$ & $1024(1.33)$ & $16448(21.39)$ \\
\hline
\end{tabular}

where $F_{t}=2^{21}+1$

Burrus $[17,22]$ have shown that for Fermat number transform with modulo base $F_{t}$ and transform length $2^{t+2}$, the generator $\alpha$ can be written as

$$
\alpha=\sqrt{2}=2^{2 t-2}\left(2^{2 t-1}-1\right)
$$

In the present case, $\alpha=\sqrt{2}=2^{48}-2^{16}$, which requires two 'ones' representation in the wordlength. Hence the multiplication of $\sqrt{2}$ requires one extra shift-add compared with the number of shift-adds for the multiplication of the single power-of-two case. However, in the fast FNT procedure (FFT-type algorithm), only one stage requires multiplications by odd powers of $\alpha$. This fact means that $\frac{1}{2}(P-1)$ extra shift-adds are used in the forward FNT transformation and $\frac{1}{4}(P-1)$ extra shift-adds for reverse FNT transformation. The resulting increase in the number of shift-adds is, of course, very small. Thus $F_{1}, F_{2}$ and $F_{3}$ form three very efficient basic sequence (transform) lengths for the computation of discrete Fourier transform using Fermat number transforms.

Returning to the sequence length problem listed in Table 1, only a few Fermat primes are suitable for the computation of DFT using FNT. However, this problem may be resolved by suitable multidimensional techniques. Consider the discrete Fourier transform length be a prime number $P$ as before. $(P-1)$ must be a composite number and let $(P-1)=N_{1} N_{2}$. The mapping from the onedimensional cyclic convolution of eqn. 7 or 8 into a twodimensional convolution can be written in linear form as follows:

$$
n=K_{1} n_{1}+K_{2} n_{2}
$$

where

$$
n_{1}=0,1, \ldots, N_{1}-1
$$

and

$$
n_{2}=0,1, \ldots, N_{2}-1
$$

Burrus [23] shows that the map is cyclic in $n_{i}$ where $i=1$ or 2 if and only if $K_{i}=C\left(N_{1} N_{2} / N_{i}\right)$, where $C$ is an integer. For a unique map, $C$ and $N_{i}$ have to be relatively prime. To apply NTT, or more precisely FNT, to effect the computation, at least one dimension of this two-dimensional mapping must be cyclic. Hence eqn. 13 is written as

$$
n=\left\langle K_{2} n_{2}+C N_{2} n_{1}\right\rangle_{P-1}
$$

where $K_{2}$ is a constant.

For this case, (i) the map is unique and cyclic in $n_{1}$ and (ii) the map is unique and not necessarily cyclic in $n_{2}$.

\section{Theorem}

The conjugate structure of the array $\left\{W_{0}^{g^{n}}: n=0,1, \ldots\right.$, $P-2\}$ is preserved in $n_{1}$ if $N_{1}$ is even for a twodimensional mapping of the array using a map $n=$ $\left\langle K_{2} n_{2}+C N_{2} n_{1}\right\rangle_{P-1}$ where $K_{2}$ and $C$ are integers and $N_{2} N_{1}=P-1$.
Before giving the proof of this theorem let us consider, for example, a 13-point DFT. Choosing $g=2$, the array $\left\{W_{0}^{g^{n}}: n=0,1, \ldots, P-2\right\}$ becomes

$$
\begin{aligned}
\left\{W_{0}^{1}, W_{0}^{2}, W_{0}^{4}, W_{0}^{8}, W_{0}^{3}, W_{0}^{6},\right. \\
\left.W_{0}^{1 *}, W_{0}^{2 *}, W_{0}^{4 *}, W_{0}^{8 *}, W_{0}^{3 *}, W_{0}^{6 *}\right\}
\end{aligned}
$$

To transform this cyclic array into a $3 \times 4$ twodimensional array, let us choose the map $n=$ $\left\langle 4 n_{2}+3 n_{1}\right\rangle_{12}$. Hence

$$
\left\{W_{0}^{g^{n}}: n=0,1, \ldots, P-2\right\} \leftrightarrow\left[\begin{array}{llll}
W_{0}^{1} & W_{0}^{8} & W_{0}^{1 *} & W_{0}^{8 *} \\
W_{0}^{3} & W_{0}^{11} & W_{0}^{3 *} & W_{0}^{1 *} \\
W_{0}^{9} & W_{0}^{7} & W_{0}^{9 *} & W_{0}^{7 *}
\end{array}\right]
$$

Hence each row (dimension $n_{1}$ ) of this resultant twodimensional array processes the same conjugate structure as the original one-dimensional array.

\section{Proof}

The map $n=\left\langle K_{2} n_{2}+C N_{2} n_{1}\right\rangle_{P-1}$ gives $N_{2}$ rows of $N_{1}$-point cyclic arrays. The term $K_{2} n_{2}$, for $n_{2}=0,1, \ldots$, $N_{2}-1$ gives a base or an offset value for finding sets of data for $N_{1}$-point cyclic arrays. Thus this term does not affect the property of the $N_{1}$-point cyclic arrays. Because the $n_{1}$ map is cyclic, $C N_{2} n_{1}$ can be written as $\left\langle C N_{2} n_{1}\right\rangle_{N_{1}}$ and actually the set of integers $\left\{\left\langle C N_{2} n_{1}\right\rangle_{N_{1}}: n_{1}=0,1, \ldots\right.$, $\left.N_{2}-1\right\}$ is merely a permutation of the set $\left\{0, N_{2}, 2 N_{2}\right.$, $\left.\ldots,\left(N_{1}-1\right) N_{2}\right\}_{\bmod P-1}$. However

$$
\begin{aligned}
& \left\{\left\langle C N_{2} n_{1}\right\rangle_{N_{1}}: n_{1}=0,1, \ldots, N_{1}-1\right\} \\
& =\left\{0, C N_{2}, \ldots, C\left(\frac{N_{1}}{2}-1\right) N_{2}, C \frac{N_{1}}{2} N_{2},\right. \\
& \left.C\left(\frac{N_{1}}{2}+1\right) N_{2}, \ldots, C\left(N_{1}-1\right) N_{2}\right\}_{\bmod N} \\
& =\left\{0, C N_{2}, \ldots, C\left(\frac{N_{1}}{2}-1\right) N_{2}, C \frac{P-1}{2},\right. \\
& \left(C \frac{P-1}{2}+C N_{2}\right), \ldots \\
& \left.\left[C \frac{P-1}{2}+C\left(\frac{N_{1}}{2}-1\right) N_{2}\right]\right\}_{\bmod P-1}
\end{aligned}
$$

From eqns. 7 and 8 , it is clear that

$$
W_{\langle n\rangle P-1}=W_{\langle n+(N / 2)\rangle P-1}^{*}
$$

Hence it can be seen from eqns. 15 and 16 that, after substituting eqn. 14 into the expression $\left\{W_{0}^{g^{n}}: n=0,1, \ldots\right.$, $P-2\}$, we get arrays

$$
\begin{array}{r}
\left\{W_{n_{2}, 0}, W_{n_{2}, 1}, \ldots, W_{n_{2},\left[\left(N_{1} / 2\right)-1\right]}, W_{n_{2}, 0}^{*},\right. \\
\left.W_{n_{2}, 1}^{*}, \ldots, W_{n_{1},\left[\left(N_{1} / 2\right)-1\right]}^{*}\right\}
\end{array}
$$

or

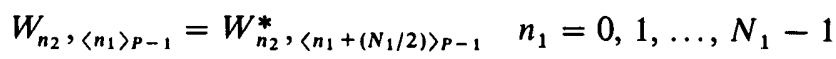

This completes the proof of the theorem.

\section{Corollary}

It is impossible to map $\left\{W_{0}^{g^{n}}: n=0,1, \ldots, P-2\right\}$ into a two-dimensional cyclic array (cyclic in both dimensions) with the conjugate structure preserved in both dimensions.

\section{Proof}

The proof of this corollary can be seen from the fact that for the mapping from a one-dimensional cyclic array into a two-dimensional cyclic array (in both dimensions) $N_{1}$ and $N_{2}$ should be relatively prime [23]. Hence either $N_{1}$ or $N_{2}$ 
can be even. From the above theorem, it is evident that for a cylic mapping of $\left\{W_{0}^{g^{n}}: n=0,1, \ldots, P-2\right\}$ in both dimensions, only one dimension preserves conjugate structure. This proves the corollary.

A direct consequence of the conjugate property of the twodimensional mapping is that the results of both real and imaginary parts of the Fermat number transformed sequence of $\left\{W_{n_{2}, n_{1}}: n_{1}=0,1, \ldots, N_{1}-1\right\}$ would be alternately zero. Hence the total number of real multiplications for each $N_{1}$-point DFT reduces from $2 N_{1}$ to $N_{1}$. Similarly, the conjugate structure of $X\left(\left\langle g^{k}\right\rangle_{P}\right)$ in eqn. 6 is also preserved for this mapping. As in the previous example, if $n=\left\langle 4 n_{2}+3 n_{1}\right\rangle_{12}$ then $k$ has to map to $\left\langle 4 n_{2}+9 n_{1}\right\rangle_{12}$ [23]. Hence we obtain

$$
\begin{aligned}
& \{X(2), X(4), X(8), X(3), X(6), X(12), \\
& \left.X^{*}(2), X^{*}(4), X^{*}(8), X^{*}(3), X^{*}(6), X^{*}(12)\right\} \\
& \leftrightarrow\left[\begin{array}{llll}
X(2) & X(10) & X^{*}(2) & X^{*}(10) \\
X(6) & X(4) & X^{*}(6) & X^{*}(4) \\
X(5) & X(12) & X^{*}(5) & X^{*}(12)
\end{array}\right]
\end{aligned}
$$

It is clear that only $X(2), X(10), X(6), X(4), X(5)$ and $X(12)$ need to be computed, the other $X(n)$ values are merely complex conjugates of these values.

Since $N_{1}$ is even, it is most worthwhile to choose $N_{1}$ as a power of two, so that the FFT-type algorithm can be used. Depending on whether the second dimension is a cylic or a linear convolution [24], pseudo-Fermat transforms, pseudo-Mersenne transforms, Mersenne number transforms, Lagrange interpolation formulas or any short convolution algorithm can be used to carry out the computation.

In practical applications, the longest transform length (with 2 or $\sqrt{2}$ as the generator) available for a Fermat number transform is 256 , but, in this case, the modulo base is $F_{6}\left(=2^{64}+1\right)$. This wordlength is too long for some computers (especially mini- or microcomputers) and in some cases in hardware implementations. Hence we begin our further discussion with a basic sequence length of 64 . With this basic sequence length, we can use any of the Fermat numbers in $\left\{F_{4}, F_{5}, F_{6}\right\}$ as the modulo base. This gives a flexible choice of wordlengths and dynamic ranges for various applications. Substituting eqns. 9 and 10 into eqn. 7 we obtain

$$
X_{k}=\sum_{n=0}^{P-2} x_{n} W_{k-n} \text { for } k=0,1, \ldots, P-2
$$

where

$$
X_{k}=X\left(\left\langle g^{k+1}\right\rangle_{P}\right)
$$

Recall $N_{1} N_{2}=P-1$. Let $N_{1}=64$ and the map of $n=$ $\left\langle K_{2} n_{2}+N_{2} n_{1}\right\rangle_{P-1}$, hence eqn. 18 becomes

$$
X_{k_{1}, k_{2}}=\sum_{n_{2}=0}^{N_{2}-1} \sum_{n_{1}=0}^{64-1} x_{n_{2}, n_{1}} W_{k_{2}-n_{2}, k_{1}-n_{1}}
$$

The map is cyclic in $n_{1}$, and so we can take the number theoretic transform of eqn. 20 with respect to $n_{1}$ [24]. Eqn. 20 becomes

$$
X_{k_{2}, k_{1}}^{\prime}=\sum_{n_{2}=0}^{N_{2}-1} X_{n_{2}, k_{1}}^{\prime \prime} W_{k_{2}-n_{2}, k_{1}}^{\prime \prime}
$$

where

$$
X_{n_{2}, k_{1}}^{\prime \prime}=\left\langle\sum_{n_{1}=0}^{64-1} x_{n_{2}, n_{1}} \alpha^{n_{1} k_{1}}\right\rangle_{M^{\prime}}
$$

$$
\begin{aligned}
& W_{k_{2}-n_{2}, k_{1}}^{\prime \prime}=\left\langle\sum_{n_{1}=0}^{64-1} W_{k_{2}-n_{2}, n_{1}} \alpha^{n_{1} k_{1}}\right\rangle_{M^{\prime}} \\
& k_{2}=0,1, \ldots, N_{2}-1,
\end{aligned}
$$

and

$$
M^{\prime}=\text { base for modulo arithmetic }
$$

Eqn. 21 is a linear convolution sum of the sequences $\left\{X_{n_{2}, k_{1}}^{\prime \prime}: n_{2}=0,1, \ldots, N_{2}-1\right\}$ and $\left\{W_{n_{2}, k_{1}}: n_{2}=0,1, \ldots\right.$, $\left.N_{2}-1\right\}$ if $N_{1}$ and $N_{2}$ are not mutually prime. This equation can be arranged as a cyclic convolution sum if $N_{1}$ and $N_{2}$ are mutually prime. For the case of linear convolutions, $\left[2-\left(1 / N_{2}\right)\right]$ [24] multiplications per point are required for the computation. These number of multiplications are still relatively large* as basic transform lengths for the application of Fermat number transform to DFT. We now turn our attention to the case with cyclic mapping in $N_{2}$. A very simple mapping is $n=\left\langle N_{1} n_{2}+N_{2} n_{1}\right\rangle_{P-1}$ where $N_{1}=64$. If $n_{2}$ is cyclic then a number theoretic transform can be used again to find this convolution sum. However, $N_{1}$ and $N_{2}$ are mutually prime, $N_{1}$ is even and we restrict our case to using FNT only, and so number theoretic transform is not suitable in this dimension.

Recall the fact that of the various short odd cyclic convolution algorithms introduced by Winograd [5] and Agarwal and Cooley [4], the length-3 cyclic convolution algorithm is the most efficient in terms of both the numbers of additions and multiplications per point needed. In fact, only four multiplications and 11 additions are required for the computation of a 3-point cyclic convolution sum. Let $N_{2}=3$ so that eqns. 20 and 21 become

$$
\begin{aligned}
& X_{k_{2}, k_{1}}=\sum_{n_{2}=0}^{3-1} \sum_{n_{1}=0}^{64-1} x_{n_{2}, n_{1}} W_{k_{2}-n_{2}, k_{1}-n_{1}} \\
& X_{k_{2}, k_{1}}^{\prime}=\sum_{n_{2}=0}^{3-1} X_{n_{2}, k_{1}}^{\prime \prime} W_{k_{2}-n_{2}, k_{1}}^{\prime \prime}
\end{aligned}
$$

where

$$
k_{1}=0,1, \ldots, 63 \quad k_{2}=0,1,2
$$

It is important to recall that the choice of $N_{1}$ and $N_{2}$ should always agree with our basic design, i.e. they must be chosen such that $(P-1)=N_{1} N_{2}$ or, equivalently, $\left(N_{1}\right.$ $\left.N_{2}+1\right)$ must be a prime number. In our case, $\left(N_{1} N_{2}+1\right)$ is equal to 193 , which is prime with 5 being the minimum primitive root.

Let us now work out the number of operations required to compute this 193-point DFT. There are 64 3-point cyclic convolutions, hence overall there are $64 \times 4=256$ multiplications. Besides the 643 -point cyclic convolutions, there are three 64-point FNT transforms (forward and reverse), hence, by using eqn. 12 , the total number of shiftadds (some are simply additions) becomes $64 \times 11+3 \times 64\left[2 \log _{2} 64+1\right]=3200$. This method requires 1.33 multiplications and 16.6 shift-adds per point respectively. Similarly, length $13(=3 \times 4+1)$-point DFT and length $97(=3 \times 32+1)$-point DFT can be computed by using $1.23 \quad(8)$ and 1.32 (14.51) multiplications (additions) per point, respectively.

On the other hand, for systems that can have relatively large word size (64-bit words for the present case), we may use a length-256 FNT with $F_{6}\left(=2^{64}+1\right)$ as a modulo base to construct another basic transform length for DFT using FNT. In this case, the DFT transform length is

* For a possible case, $N_{2}=2$, yielding 1.5 multiplications to compute one point Thus this is still a practical case, but the number of multiplications per point is still slightly more than the number of multiplications per point to be discussed below. 
$(256 \times 3+1)=769$, which is a prime number again, with 11 being the minimum primitive root. The total number of multiplications is $256 \times 4=1024$ and the total number of shift-adds becomes $256 \times 11+3 \times 4544=16448$. The DFT with this sequence length requires 1.33 multiplications and 21.4 shift-adds per point, respectively.

Length 3-, 5-, 17-, 257-, 13-, 97-, 193- and 769-point DFTs form eight basic Fermat number transform modules, and these modules are used to construct algorithms for computing long discrete Fourier transforms in the next Section.

\section{Prime factor implementation}

The basic idea in this Section arises mainly from the fact that all eight basic Fermat number transform modules obtained so far are prime in length. Thus any two or more of these modules can form basic lengths for two- or multidimensional rearrangements of discrete Fourier transforms. Furthermore, any one or more of these modules can also combine with other short (or long if there happen to be any) DFT algorithms for computing long discrete Fourier transforms.

Now let us examine the multidimensional mapping of the DFT equation. For the sake of simplicity, we consider a simple two-dimensional case. Let $N$ of eqn. 4 be a composite number, $N_{3} N_{4}=N$. Further, let $N_{3}$ and $N_{4}$ be relatively prime so that the 'twiddle factors' [25] of the multidimensionally rearranged DFT equation can be avoided by the application of the Chinese remainder theorem (CRT) $[26,23]$. In this case eqn. 4 can be written as

$$
Y_{k_{4}, k_{3}}=\sum_{n_{4}=0}^{N_{4}-1} \sum_{n_{3}=0}^{N_{3}-1} x_{n_{4}, n_{3}} W_{3}^{n_{3} k_{3}} W_{4}^{n_{4} k_{4}}
$$

where

$$
\begin{aligned}
& W_{3}=\exp \left(-j \frac{2 \pi}{N_{3}}\right) \\
& W_{4}=\exp \left(-j \frac{2 \pi}{N_{4}}\right)
\end{aligned}
$$

and the mapping becomes

$$
\begin{aligned}
& n=N_{3} n_{4}+N_{4} n_{3} \\
& k=N_{3} N_{3}^{-1} k_{4}+N_{4} N_{4}^{-1} k_{3}
\end{aligned}
$$

where

$$
\begin{aligned}
& \left\langle N_{3} N_{3}^{-1}\right\rangle_{N_{4}}=1 \\
& \left\langle N_{4} N_{4}^{-1}\right\rangle_{N_{3}}=1
\end{aligned}
$$

Recall also the fact that both the prime factor algorithm (PFA) [6] and the Winograd-Fourier-transform algorithm [5] essentially make use of the same multidimensional mapping and short DFT algorithms for their computation. The number of operations per point becomes rather large for increasing DFT sizes. This is mainly due to the nature of multidimensional mapping and also due to limited number of available efficient short DFTs. There are mainly eight, namely length-2, 3, 4, 5, 7, 8, 9, 16 efficient short DFT algorithms [3, 9]. Four of these are powers of two; hence only one of the these four can appear in the same multidimensional rearranged DFT. However, if we add our basic FNT modules to these eight short DFT algorithms we have available a much larger choice in transform lengths, and the number of multiplications per point remains a very small number even for DFT lengths longer than 10000 .
Eqn. 26 can now be written in the following form suitable for a prime factor algorithm [6],

$$
Y_{k_{4}, k_{3}}=\sum_{n_{4}=0}^{N_{4}-1}\left(\sum_{n_{3}=0}^{N_{3}-1} x_{n_{4}, n_{3}} W_{3}^{n_{3} k_{3}}\right) W_{4}^{n_{4} k_{4}}
$$

The two-dimensional transform in eqn. 29 may be implemented by first calculating $N_{4}$ length- $N_{3}$ DFTs

$$
Y_{n_{4}, k_{3}}^{\prime}=\sum_{n_{3}=0}^{N_{3}-1} x_{n_{4, n_{3}}} W_{3}^{n_{3} k_{3}}
$$

then calculating $N_{3}$ length- $N_{4}$ DFTs

$$
Y_{k_{1}, k_{2}}=\sum_{n_{4}=0}^{N_{4}-1} Y_{n_{4}, k_{3}}^{\prime} W_{4}^{n_{4} k_{4}}
$$

The number of multiplications and additions become

$$
\begin{aligned}
& M=N_{4} M_{3}+N_{3} M_{4} \\
& A=N_{4} A_{3}+N_{3} A_{4}
\end{aligned}
$$

where $M_{3}\left(A_{3}\right)$ and $M_{4}\left(A_{4}\right)$ are the number of multiplications (additions) for lengths $N_{3}$ and $N_{4}$ DFTs, respectively. Any one of our eight FNT modules can be used in $N_{3}$-point DFTs, and any one of the eight short DFTs can be used in $N_{4}$-point DFTs, provided that $N_{3}$ and $N_{4}$ are relatively prime. However, on close examination of the eight short DFT algorithms it becomes clear that the most efficient lengths are 2 and 4 , in that both require trivial multiplications only. In this case eqn. 32 becomes $M=$ $N_{4} M_{3}$. This immediately doubles or quadruples the sequence lengths of the four FNT modules without increasing the number of required multiplications per point. For other transform lengths there is an unavoidable but nevertheless slight increase in the number of multiplications per point.

Eqn. 26 may also be described by the following form suitable for computation using the Winograd-Fouriertransform algorithm [5]:

$$
\bar{Y}_{k_{4}}=\sum_{n_{4}=0}^{N_{4}-1}\left(\bar{W} \bar{X}_{n_{4}}\right) W_{4}^{n_{4} k_{4}}
$$

where

$$
\begin{gathered}
\bar{X}_{n_{4}}=\left[\begin{array}{l}
x_{n_{4}, 0} \\
x_{n_{4}, 1} \\
\cdots \\
\ldots
\end{array}\right] \quad \bar{Y}_{k_{4}}=\left[\begin{array}{l}
Y_{k_{4}, 0} \\
Y_{k_{4}, 1} \\
\cdots \\
\cdots \\
x_{n_{4}, N_{3}-1}
\end{array}\right] \\
\bar{W}=\left[\begin{array}{llll}
W_{3}^{0} & W_{3}^{0} & \cdots & W_{3}^{0} \\
W_{3}^{0} & W_{3}^{1} & \cdots & W_{3}^{N_{3}-1} \\
\cdots & \cdots & \cdots & \cdots \\
\cdots & \cdots & \cdots & \cdots \\
W_{3}^{0} & \cdots & \cdots & W_{3}^{\left(N_{3}-1\right)^{2}}
\end{array}\right]
\end{gathered}
$$

Eqn. 34 is obtained by considering eqn. 26 as essentially a one-dimensional DFT of length $N_{4}$ with each scalar replaced by a vector of $N_{3}$ terms. The number of operations in this case becomes

$$
\begin{aligned}
& M=M_{1} M_{2} \\
& A=N_{1} A_{2}+M_{2} A_{1}
\end{aligned}
$$

However, owing to the nesting nature of this algorithm, the trivial multiplications (the multiplications of $W^{0}$ ) in the basic FNT modules cannot be ignored in eqn. 35. From eqns. 5 and 6 it is clear that there are two trivial multiplications, namely $\left(\sum_{n=0}^{P-1} x(n)\right) W_{0}^{0}$ and $x(0) W_{0}^{0}$. To make a 
slight simplification of the computation, let us make a rearrangement of eqn. 6

$$
Y\left(\left\langle g^{k}\right\rangle_{P}\right)=x(0)+\sum_{n=1}^{P-1} x\left(\left\langle g^{-n}\right\rangle_{P}\right) W_{0}^{\left\langle g^{k-n}\right\rangle_{P}}
$$

such that

$$
Y\left(\left\langle g^{k}\right\rangle_{P}\right)=\sum_{n=1}^{P-1}\left[x\left(\left\langle g^{-n}\right\rangle_{P}\right)-x(0)\right] W_{0}^{\left\langle g^{k-n}\right\rangle_{P}}
$$

Hence, if eqn. 37 is used instead of eqn. 6, one $W_{0}^{0}$ multiplication is saved. The only difference between eqn. 6 and eqn. 37 is that $x(0)$ is included before the computation of the cyclic convolution in eqn. 37 whereas $x(0)$ is included after the computation of the cyclic convolution in eqn. 6 .

As shown in Table 2, if we include the multiplication of $\left(\sum_{n=0}^{P=1} x(n)\right] W_{0}^{0}$ in eqn. 5 , the number of multiplications

Table 2: Number of multiplications (including the multiplications of $W_{0}^{0}$ ) for eight basic FNT modules

\begin{tabular}{lc}
\hline $\begin{array}{l}\text { DFT } \\
\text { length }\end{array}$ & $\begin{array}{l}\text { Number of } \\
\text { multiplications } \\
N\end{array}$ \\
\hline 3 & $M_{3}$ \\
\hline 5 & 3 \\
17 & 17 \\
257 & 257 \\
13 & 17 \\
97 & 129 \\
193 & 257 \\
769 & 1025 \\
\hline
\end{tabular}

required for the eight basic FNT modules increases slightly.

Let us illustrate this idea with $N=15$. Hence $N=15=3 \times 5$. Eqn. 27 becomes

$$
n=\left\langle 5 n_{4}+3 N_{3}\right\rangle_{15}
$$

where

$$
\begin{aligned}
& n_{3}=0,1,2,3,4 \\
& n_{4}=0,1,2
\end{aligned}
$$

i.e.

$$
\begin{array}{lll}
x_{0,0}=x(0) & x_{1,0}=x(5) & x_{2,0}=x(10) \\
x_{0,1}=x(3) & x_{1,1}=x(8) & x_{2,1}=x(13) \\
x_{0,2}=x(6) & x_{1,2}=x(11) & x_{2,2}=x(1) \\
x_{0,3}=x(9) & x_{1,3}=x(14) & x_{2,3}=x(4) \\
x_{0,4}=x(12) & x_{1,4}=x(2) & x_{2,4}=x(7)
\end{array}
$$

Eqns. 26 and 34 become

$$
Y_{k_{4}, k_{3}}=\sum_{n_{4}=0}^{3-1}\left(\sum_{n_{3}=0}^{5-1} x_{n_{4}, n_{3}} W_{3}^{n_{3} k_{3}}\right) W_{4}^{n_{4} k_{4}}
$$

and

$$
\bar{Y}_{k_{4}}=\sum_{n_{4}=0}^{2}\left(\bar{W} \bar{X}_{n 4}\right) W_{4}^{n_{4} k_{4}}
$$

where

$$
\bar{Y}_{k_{4}}=\left[\begin{array}{l}
Y_{k_{4}, 0} \\
Y_{k_{4}, 1} \\
Y_{k_{4}, 2} \\
Y_{k_{4}, 3} \\
Y_{k_{4}, 4}
\end{array}\right] \quad \bar{X}_{n_{4}}=\left[\begin{array}{l}
x_{n_{4}, 0} \\
x_{n_{4}, 1} \\
x_{n_{4}, 2} \\
x_{n_{4}, 3} \\
x_{n_{4}, 4}
\end{array}\right]
$$

$$
\bar{W}=\left[\begin{array}{lllll}
W_{3}^{0} & W_{3}^{0} & W_{3}^{0} & W_{3}^{0} & W_{3}^{0} \\
W_{3}^{0} & W_{3}^{1} & W_{3}^{2} & W_{3}^{3} & W_{3}^{4} \\
W_{3}^{0} & W_{3}^{2} & W_{3}^{4} & W_{3}^{1} & W_{3}^{5} \\
W_{3}^{0} & W_{3}^{3} & W_{3}^{1} & W_{3}^{4} & W_{3}^{2} \\
W_{3}^{0} & W_{3}^{4} & W_{3}^{3} & W_{3}^{2} & W_{3}^{1}
\end{array}\right]
$$

and

$$
W_{3}=\exp \left(-j \frac{2 \pi}{5}\right) \text { for } n_{4}=0,1,2
$$

Now we may use the 3-point DFT algorithm (see Reference 5 , for example), to evaluate eqn. 38

$$
\begin{aligned}
\bar{S}_{1} & =\bar{X}_{1}+\bar{X}_{2} \\
\bar{S}_{2} & =\bar{X}_{1}-\bar{X}_{2} \\
\bar{S}_{0} & =\bar{X}_{0}+\bar{S}_{1} \\
\bar{M}_{0} & =1 \cdot \bar{W} \bar{S}_{0} \\
\bar{M}_{1} & =\left(\cos \frac{-2 \pi}{3}-1\right) \cdot \bar{W} \bar{S}_{1} \\
\bar{M}_{2} & =j \sin \frac{-2 \pi}{3} \cdot \bar{W} \bar{S}_{2} \\
\bar{S}_{3} & =\bar{M}_{0}+\bar{M}_{1} \\
\bar{S}_{4} & =\bar{S}_{3}+\bar{M}_{2} \\
\bar{S}_{5} & =\bar{S}_{3}-\bar{M}_{2}
\end{aligned}
$$

and

$$
\begin{aligned}
& \bar{Y}_{0}=\bar{M}_{0} \\
& \bar{Y}_{1}=\bar{S}_{4} \\
& \bar{Y}_{2}=\bar{S}_{5}
\end{aligned}
$$

All three expressions in eqn. 39 are actually length-5 DFTs multiplied by constants and are in the following form

$$
\bar{M}_{k}=\operatorname{CON} \bar{W} \bar{S}_{k}
$$

where $C O N$ is a constant and

$$
k=0,1,2
$$

Let

$$
\bar{M}_{k}=\left[\begin{array}{l}
m_{k, 0} \\
m_{k, 1} \\
m_{k, 2} \\
m_{k, 3} \\
m_{k, 4}
\end{array}\right] \text { and } \bar{S}_{k}=\left[\begin{array}{c}
s_{k, 0} \\
s_{k, 1} \\
s_{k, 2} \\
s_{k, 3} \\
s_{k, 4}
\end{array}\right]
$$

Using the Fermat number transform, we obtain

$$
\left[\begin{array}{l}
m_{k, 0} \\
m_{k, 2} \\
m_{k, 4} \\
m_{k, 3} \\
m_{k, 1}
\end{array}\right]=\operatorname{CON}\left[\begin{array}{l}
W_{3}^{0}\left(s_{k, 0}+s_{k, 1}+s_{k, 2}+s_{k, 3}+s_{k, 4}\right) \\
b_{k, 2} \\
b_{k, 4} \\
b_{k, 3} \\
b_{k, 1}
\end{array}\right]
$$

where

$$
\left[\begin{array}{l}
b_{k, 2} \\
b_{k, 4} \\
b_{k, 3} \\
b_{k, 1}
\end{array}\right]=\frac{1}{4}\left[\begin{array}{cccc}
1 & 1 & 1 & 1 \\
1 & \alpha^{-1} & \alpha^{-2} & \alpha^{-3} \\
1 & \alpha^{-2} & \alpha^{0} & \alpha^{-2} \\
1 & \alpha^{-3} & \alpha^{-2} & \alpha^{-1}
\end{array}\right]\left\{\left[\begin{array}{cccc}
1 & 1 & 1 & 1 \\
1 & \alpha^{1} & \alpha^{2} & \alpha^{3} \\
1 & \alpha^{2} & \alpha^{0} & \alpha^{2} \\
1 & \alpha^{3} & \alpha^{2} & \alpha^{1}
\end{array}\right]\right.
$$

IEE PROCEEDINGS, Vol. 131, Part F, No. I, FEBRUARY 1984 


$$
\left.\times\left[\begin{array}{l}
W_{3}^{1} \\
W_{3}^{2} \\
W_{3}^{4} \\
W_{3}^{3}
\end{array}\right] \otimes\left[\begin{array}{cccc}
1 & 1 & 1 & 1 \\
1 & \alpha^{1} & \alpha^{2} & \alpha^{3} \\
1 & \alpha^{2} & \alpha^{0} & \alpha^{2} \\
1 & \alpha^{3} & \alpha^{2} & \alpha^{1}
\end{array}\right]\left[\begin{array}{c}
\left(s_{k, 3}-s_{k, 0}\right) \\
\left(s_{k, 4}-s_{k, 0}\right) \\
\left(s_{k, 2}-s_{k, 0}\right) \\
\left(s_{k, 1}-s_{k, 0}\right)
\end{array}\right]\right\}
$$

where $\otimes$ means term-by-term multiplication.

Notice that, for an actual implementation, all coefficients must be scaled to integers and they must be within the allowable dynamic range $[17,18]$. The $C O N$ in eqn. 40 and ' $1 / 4$ ' in eqn. 41 should be absorbed inside the $W_{2} \mathrm{~s}$, in which case no extra multiplication is required, and actually this is the original idea of the Winograd-Fourier-transform algorithm.

For our discussion so far, we have combined one of our basic FNT modules with one short DFT algorithm. However, in general, one may choose to combine the FNT modules to form a long transform or indeed any number of short DFT or FNT modules can be combined to form long DFT transforms using the prime factor algorithm or the Winograd-Fourier-transform algorithm. The only restriction is that the sequence lengths of these short DFT algorithms and FNT modules must be mutually prime.

\section{Hybrid implementation}

It is seen that both the prime factor algorithm and the Winograd-Fourier-transform algorithm can combine with eight FNT modules to compute long DFTs. PFA is especially efficient for sequence lengths of 2 and 4 . WFTA is very efficient for lengths 3 and 8 , hence it is worthwhile to use a combination of these methods to compute very long DFT sequences.

Table 3 lists the number of real multiplications and real

Table 3: Number of operations per point for DFTs computed by FNT with modulo base $F_{4}, F_{5}$ or $F_{6}$

\begin{tabular}{llll}
\hline $\begin{array}{l}\text { DFT } \\
\text { length }\end{array}$ & $\begin{array}{l}\text { Prime } \\
\text { factors }\end{array}$ & $\begin{array}{l}\text { Number of } \\
\text { multiplications } \\
\text { per point }\end{array}$ & $\begin{array}{l}\text { Number of } \\
\text { shift-adds } \\
\text { per point }\end{array}$ \\
\hline 17 & 17 & 0.94 & 8.47 \\
34 & $2 \times 17$ & 0.94 & 9.47 \\
68 & $4 \times 17$ & 0.94 & 10.47 \\
102 & $2 \times 3 \times 17$ & 1.00 & 11.47 \\
136 & $8 \times 17$ & 1.00 & 11.72 \\
204 & $3 \times 4 \times 17$ & 1.00 & 12.47 \\
408 & $3 \times 8 \times 17$ & 1.00 & 13.72 \\
680 & $5 \times 8 \times 17$ & 1.00 & 15.72 \\
1020 & $3 \times 4 \times 5 \times 17$ & 1.00 & 16.47 \\
2040 & $3 \times 5 \times 8 \times 17$ & 1.00 & 17.72 \\
4080 & $3 \times 5 \times 16 \times 17$ & 1.13 & 19.10 \\
14280 & $3 \times 5 \times 7 \times 8 \times 17$ & 1.29 & 22.86 \\
23160 & $3 \times 5 \times 8 \times 193$ & 1.33 & $25.83 * *$ \\
39576 & $3 \times 8 \times 17 \times 97$ & 1.33 & 28.24 \\
78744 & $3 \times 8 \times 17 \times 193$ & 1.33 & $30.30^{* *}$ \\
\hline
\end{tabular}

** An additional 0.75 shift-add per point is required for modulo $F_{4}$

additions for various DFTs computed by this hybrid technique with 5-point, 17-point, 97-point and 193-point FNT modules. In this case either $F_{4}$ or $F_{5}$ or $F_{6}$ can be chosen as the modulo base for the computation, and this results in a flexible choice in word lengths for the implementation. Figs. 1 and 2 give the number of multiplications and additions for WFTA [5], polynomial transform (PT) [9] and for the present DFT computation technique. As can be seen from these Figures, the number of multiplications for WFTA and the polynomial transform is between 30 and $90 \%$ higher than the corresponding number of multiplica- tions for the present method. On the other hand, the present method requires between 20 to $30 \%$ more shift-

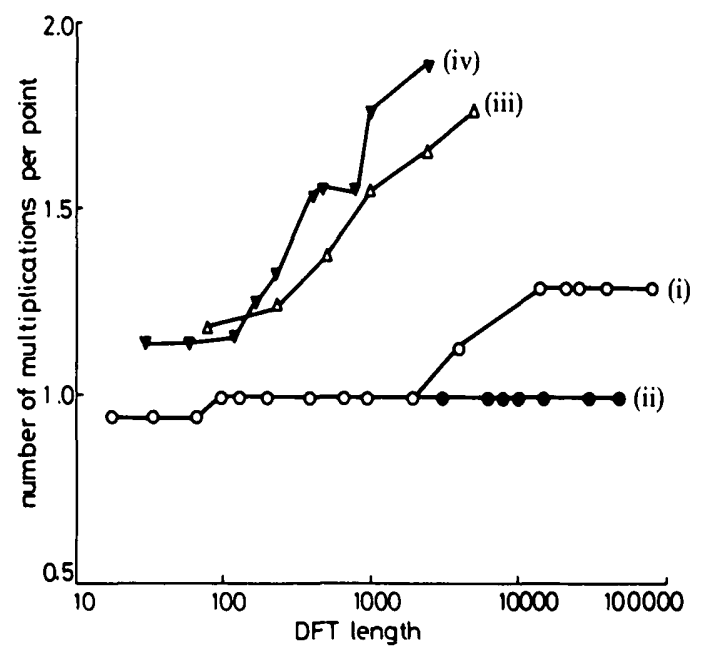

Fig. 1 Number of multiplications per point against sequence length for DFTs

(i) computed by FNT with modulo base $F_{4}, F_{5}$ or $F_{6}(O)$

(ii) computed by FNT with modulo base $F_{6}$ only (O)

(iii) computed by polynomial transform $(\Delta)$

(iv) computed by Winograd-Fourier-transform algorithm ( $\nabla)$

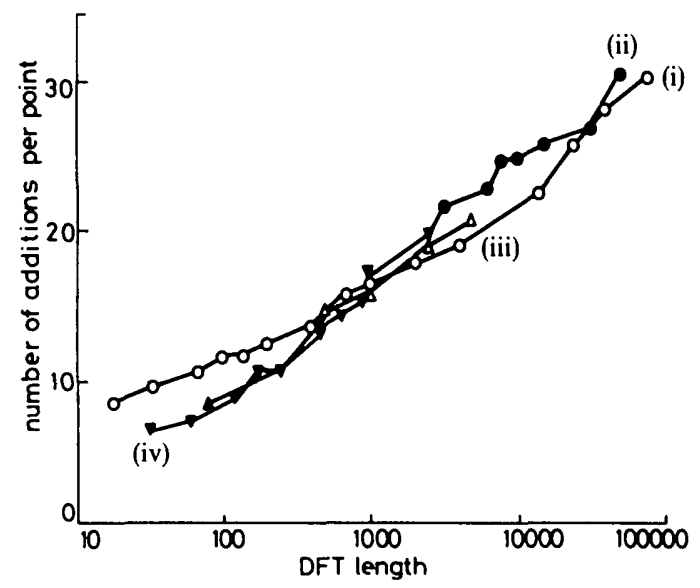

Fig. 2 Number of shift-adds (or additions) per point against sequence length for DFTs

(i) computed by FNT with modulo base $F_{4}, F_{3}$ or $F_{6}(O)$

(ii) computed by FNT with modulo base $F_{6}$ only (O)

(iii) computed by polynomial transform $(\triangle)$

(iv) computed by Winograd-Fourier-transform algorithm ( $\nabla)$

adds than the number of additions in WFTA and polynomial transforms for short sequence lengths. However, for sequence length 680 or above, the number of shift-adds for the present method is equal to or less than the number of additions for WFTA and PT. Hence the present method is more useful for long DFT lengths. It is worth pointing out that, for dedicated hardware or microcomputer implementation, the multiplication time is of the order of ten times or more slower than addition, notwithstanding the relative costs of such operations.

Table 4 lists the number of real operations required for various DFT using the 257-point FNT module. In this case, $F_{6}$ has to be used as the modulo base for the computation. It is clear from Fig. 1 that the number of multiplications per point remains constant and is equal to unity for even extremely long DFT sequences. Hence the present method gives a very efficient way to compute long DFTs.

\section{Conclusion}

Eight basic FNT modules for computing DFTs have been presented and used as the basic transform lengths to 
Table 4: Number of operations per point for DFTs computed by FNT with modulo base $F_{6}$ only

\begin{tabular}{llll}
\hline $\begin{array}{l}\text { DFT } \\
\text { length }\end{array}$ & $\begin{array}{l}\text { Prime } \\
\text { factors }\end{array}$ & $\begin{array}{l}\text { Number of } \\
\text { multiplications } \\
\text { per point }\end{array}$ & $\begin{array}{l}\text { Number of } \\
\text { shift-adds } \\
\text { per point }\end{array}$ \\
\hline 257 & 257 & 1.00 & 17.7 \\
514 & $2 \times 257$ & 1.00 & 18.7 \\
1028 & $4 \times 257$ & 1.00 & 19.7 \\
1542 & $2 \times 3 \times 257$ & 1.00 & 20.7 \\
2056 & $8 \times 257$ & 1.00 & 20.9 \\
3084 & $3 \times 4 \times 257$ & 1.00 & 21.7 \\
6168 & $3 \times 8 \times 257$ & 1.00 & 22.9 \\
7710 & $2 \times 3 \times 5 \times 257$ & 1.00 & 24.7 \\
10280 & $5 \times 8 \times 257$ & 1.00 & 24.9 \\
15420 & $3 \times 4 \times 5 \times 257$ & 1.00 & 25.7 \\
30840 & $3 \times 5 \times 8 \times 257$ & 1.00 & 26.9 \\
52428 & $3 \times 4 \times 17 \times 257$ & 1.00 & 30.2 \\
\hline
\end{tabular}

compute long discrete Fourier transforms. The number of multiplications per point for most cases is not more than one and the number of shift-adds is almost equal to (for long sequence lengths, less than) the number of additions in the Winograd-Fourier-transform algorithm and the polynomial transform. Thus the present technique is very effective in computing discrete Fourier transforms.

Some possible transform lengths based on the eight basic FNT modules have been listed, but this listing is no means exhaustive in that the user may combine different short DFTs to form other transform lengths as required. Hence this method gives a large class of transform lengths for computing discrete Fourier transforms.

Fermat number transforms have already been used to compute convolutions effectively. These are mainly implemented through dedicated hardware or modules (with add-on hardware sometimes) written in assembly language for a computer/microcomputer. Hence, with just some slight modifications of these modules, one may compute a discrete Fourier transform using FNT on the same system. However, since most computer/microcomputer hardware is not designed to perform modulo Fermat number arithmetic, it is perhaps desirable to fabricate digital signalprocessor chips with FNT arithmetic which can facilitate the computation of DFT and convolution using FNT. The major problem left to be solved is concerned with the design of the architecture of the digital signal processor.

\section{Acknowledgment}

The authors wish to thank the referees for their helpful comments and suggestions.

\section{References}

1 CAPPELliNI, V., CONSTANTINIDES, A.G., and EMILIANI, P.: 'Digital filters and their applications' (Academic Press, 1978)
2 COOLEY, J.W., and TUKEY, J.W.: 'An algorithm for the machine calculation of complex Fourier series', Math. Comput., 1965, 19, pp. 297-301

3 WINOGRAD, S.: 'Some bilinear forms whose multiplicative complexity depends on the field of constants', IBM T.J. Watson Research Centre, New York, USA, IBM Res. Rep. RC5669, 1975

4 AGARWAL, R.C., and COOLEY, J.W.: 'New algorithms for digital convolution', IEEE Trans., 1977, ASSP-25, pp. 106-124

5 WINOGRAD, S.: 'On computing the discrete fourier transform', Math. Comput., 1978, 32, pp. 175-199

6 KOLBA, D.P., and PARKS, T.W.: 'A prime factor FFT algorithm using high-speed convolution', IEEE Trans., 1977, ASSP-25, pp. 91-103

7 RADER, C.M.: 'Discrete Fourier transforms when the number of data samples is prime', Proc. IEEE, 1968, 56, pp. 1107-1108

8 NUSSBAUMER, H.J.: 'Digital filtering using polynomial transforms', Electron. Lett., 1977, 13, (13), pp. 386-387

9 NUSSBAUMER, H.J., and QUANDALLE, P.: 'Fast convolution of discrete Fourier transforms using polynomial transforms', IEEE Trans., 1979, ASSP-27, pp. 169-181

10 NUSSBAUMER, H.J., and QUANDALLE, P.: 'Computation of convolution and discrete Fourier transforms by polynomial transforms', IBM J. Res. \& Dev., 1978, 22, pp. 134-144

11 NUSSBAUMER, H.J.: 'New polynomial transform algorithms for multi-dimensional DFT's and convolutions', IEEE Trans., 1981, ASSP-29, pp. 74-83

12 REED, I.S., and TRUONG, T.K.: 'A fast D.F.T. algorithm using complex integer transforms', Electron. Lett., 1978, 14, (6), pp. 191-193

13 REED, I.S., and TRUONG, T.K.: 'A new hybrid algorithm for computing a fast discrete Fourier transform', IEEE Trans., 1979, C-28, pp. 487-492

14 SIU, W.C., and CONSTANTINIDES, A.G.: 'Very fast discrete Fourier transform'. IEE Seminar on Digital Filters, London, 7th-8th April 1983, pp. 1-29

15 POLLARD, J.M.: 'The fast Fourier transform in a finite field', Math. Comput., 1971, 25, pp. 365-374

16 RADER, C.M.: 'Discrete convolution via Mersenne transform', IEEE Trans., 1972, C-21, pp. 1269-1273

17 AGARWAL, R.C., and BURRUS, C.S.: 'Fast convolution using Fermat number transform with application to digital filtering', ibid., 1974, ASSP-22, pp. 87-97

18 SIU, W.C.: 'Number theoretic transform and its applications to digital signal processing'. Proc., IERE Hong Kong Section Workshop on Adv. Micro. \& DSP, Hong Kong, Sept. 1982, pp. 76-101

19 NUSSBAUMER, H.J.: 'Digital filtering using pseudo Fermat number transform', IEEE Trans., 1977, ASSP-26, pp. 79-83

20 NUSSBAUMER, H.J.: 'Digital filtering using complex Mersenne transforms', IBM J. Res. \& Dev., 1976, 20, pp. 498-504

21. RADER, C.M.: 'On the application of number theoretic methods of high speed convolution to two-dimensional filtering', IEEE Trans., 1975, CAS-22, p. 575

22 DICKSON, L.E.: 'History of the theory of numbers', (Carnegie Institute, 1919)

23 BURRUS, C.S.: 'Index mapping for multi-dimensional formulation of the DFT and convolution', IEEE Trans., 1977, ASSP-25, pp. 239-242

24 SIU, W.C., and CONSTANTINIDES, A.G.: 'On the cyclic convolution of long sequences using number theoretic transform'. Research Report, EE, Imperial College of Science \& Technology, Jan. 1983

25 GENTLEMAN, W.M., and SANDE, G.: 'Fast Fourier transforms for fun and profit'. 1966 Fall Joint Comput. Conf., AFIPS Proc., Vol. 29, Washington, DC, (Spartan, 1966), pp. 563-578

26 GOOD, I.J.: 'The relationship between two fast Fourier transforms', IEEE Trans., 1971, C-20, pp. 310-317 